CONFORMAL GEOMETRY AND DYNAMICS

An Electronic Journal of the American Mathematical Society

Volume 5, Pages 74-80 (August 14, 2001)

S $1088-4173(01) 00074-1$

\title{
TRANSVERSELY PROJECTIVE STRUCTURES ON A TRANSVERSELY HOLOMORPHIC FOLIATION
}

\author{
INDRANIL BISWAS
}

\begin{abstract}
The space of transversely projective structures on a transversely
\end{abstract} holomorphic foliation is described. Some applications are given.

\section{INTRODUCTION}

Let $\mathcal{F}$ be a codimension two foliation on a $C^{\infty}$ manifold $M$. A transversely holomorphic foliation on $\mathcal{F}$ is defined by data of the following type:

Let $\left\{U_{i}\right\}_{i \in I}$ be an open covering of $M$, and let $\phi_{i}: U_{i} \longrightarrow \mathbb{C}$ be submersions onto the image such that $\mathcal{F}$ is the kernel of the differential $d \phi_{i}$, or in other words, the fibers of $\phi_{i}$ are leaves for $\mathcal{F}$. If there is a biholomorphic map

$$
f_{i, j}: \phi_{i}\left(U_{i} \cap U_{j}\right) \longrightarrow \phi_{j}\left(U_{i} \cap U_{j}\right)
$$

for each pair $i, j \in I$ such that the following diagram is commutative

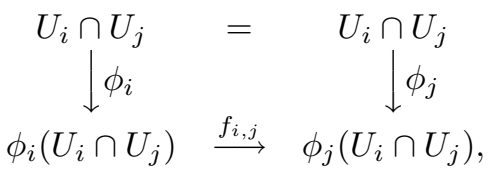

then $\left\{U_{i}, \phi_{i}, f_{i, j}\right\}$ defines a transversely holomorphic structure on $\mathcal{F}$.

A projective structure on a Riemann surface $X$ is defined by giving a covering of $X$ by holomorphic coordinate charts such that all the transition functions are restrictions of Möbius transformations.

The notion of a projective structure can be extended to the situation of foliations. We recall that a transversely holomorphic foliation $\mathcal{F}$ as above is a transversely projective foliation if each $f_{i, j}$ is the restriction of a Möbius transformation to $\phi_{i}\left(U_{i} \cap U_{j}\right)$.

Let $\mathcal{F}$ be a transversely holomorphic foliation. The normal bundle $N:=T M / \mathcal{F}$ has a Bott connection, as explained in Section 2. Moreover, it has a holomorphic structure in the transverse direction in a sense which is briefly described below (the details are given in Section 2).

The pullback, using the above maps $\phi_{i}$, of anti-holomorphic differentials on $\mathbb{C}$ define a $C^{\infty}$ line bundle $\overline{N^{*}}$ over $M$ equipped with a Bott connection. The holomorphic structure on $N$ in the transverse direction is a homomorphism from (locally defined) flat sections (with respect to the Bott partial connection) of $N$ to the flat sections of $\overline{N^{*}} \otimes N$ with respect to the Bott partial connection satisfying a Leibniz

Received by the editors February 4, 2001 and, in revised form, July 9, 2001.

2000 Mathematics Subject Classification. Primary 37F75.

(C)2001 American Mathematical Society 
identity. The holomorphic structure on $N$ in the transverse direction induces a holomorphic structure in the transverse direction on any tensor power of $N$.

We prove that the space of transversely projective structures on $\mathcal{F}$ (with the transversely holomorphic structure fixed) is an affine space for the space of global sections of $N^{*} \otimes N^{*}$ that are both flat with respect to the Bott partial connection and holomorphic with respect to the transversely holomorphic structure of $N^{*} \otimes N^{*}$ (Theorem 2.6).

Let $M$ be a compact Kähler manifold and $\mathcal{F}$ be a holomorphic foliation of codimension one. As a corollary of Theorem 2.6 we prove that if the holomorphic tangent bundle $T_{M}$ is semistable of positive degree, then there can be at most one transversely projective structure on $\mathcal{F}$ (Lemma 2.7).

\section{TRANSVERSELY hOLOMORPHiC FOLIATIONS}

Let $M$ be a connected smooth real manifold of dimension $d+2$. Let $\mathcal{F}$ be a $C^{\infty}$ subbundle of rank $d$ of the tangent bundle $T M$.

We will recall the definition of a transversely holomorphic structure.

Definition 2.1. A transversely holomorphic structure on $\mathcal{F}$ is defined by giving the following data [4], 2]:

(1) A covering of $M$ by open subsets $U_{i}$, where $i$ runs over an index set $I$. Hence we have $\bigcup_{i \in I} U_{i}=M$.

(2) For each $i \in I$, a submersion $\phi_{i}$ of $U_{i}$ to an open subset $D_{i}$ of $\mathbb{C}$. The restriction $\left.\mathcal{F}\right|_{U_{i}}$ is the kernel of the differential map $d \phi_{i}: T U_{i} \longrightarrow \phi_{i}^{*} T D_{i}$.

(3) For every pair $i, j \in I$, there is a commutative diagram of maps

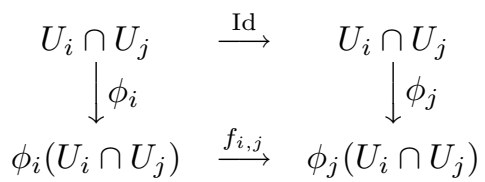

where $f_{i, j}$ is a holomorphic map.

Note that the second condition implies that $\mathcal{F}$ is closed under Lie bracket, or in other words, $\mathcal{F}$ is a foliation.

Two such data $\left\{U_{i}, \phi_{i}\right\}_{i \in I}$ and $\left\{U_{i}, \phi_{i}\right\}_{i \in J}$ are called equivalent if their union, namely

$$
\left\{U_{i}, \phi_{i}\right\}_{i \in I \cup J},
$$

also satisfies the above conditions. A transversely holomorphic structure on $\mathcal{F}$ will mean an equivalence class of data of the above type satisfying the above three conditions.

Next we will recall the definition of a transversely projective foliation. Let $\mathcal{F}$, as before, be a $C^{\infty}$ subbundle of rank $d$.

Definition 2.2. A transversely projective structure on $\mathcal{F}$ is defined by giving a data $\left\{U_{i}, \phi_{i}\right\}_{i \in I}$ exactly as in Definition 2.1, but satisfying the extra condition (apart from the three conditions in Definition 2.1) that the holomorphic maps $f_{i, j}$ in condition (3) are of the form $z \longmapsto(a z+b) /(c z+d)$, where $a, b, c, d \in \mathbb{C}$ are constant scalars and $a d-b c=1$, or in other words, each $f_{i, j}$ is the restriction of some Möbius transformation. The scalars $a, b, c, d$ may depend on the index $i$. As before, two such data $\left\{U_{i}, \phi_{i}\right\}_{i \in I}$ and $\left\{U_{i}, \phi_{i}\right\}_{i \in J}$ are called equivalent if their union 
$\left\{U_{i}, \phi_{i}\right\}_{i \in I \cup J}$ is also a data for a transversely projective structure. A transversely projective structure on $\mathcal{F}$ will mean an equivalence class of such data.

Clearly, a transversely projective structure on $\mathcal{F}$ defines a transversely holomorphic structure on $\mathcal{F}$. If $\overline{\mathcal{F}}$ is a transversely holomorphic structure on $\mathcal{F}$, then a transversely projective structure on $\overline{\mathcal{F}}$ is a transversely projective structure on $\mathcal{F}$ such that the transversely holomorphic structure defined by it coincides with $\overline{\mathcal{F}}$.

If $d=0$, then a transversely holomorphic structure on $\mathcal{F}$ is simply a complex structure on the surface $M$, and a transversely projective structure on $\mathcal{F}$ is a projective structure on $M$.

Any two projective structures on a Riemann surface $C$ differ by a quadratic differential on $C$, that is, a holomorphic section of $K_{C}^{\otimes 2}$, where $K_{C}:=T^{*} C$ is the holomorphic cotangent bundle. More precisely, the space of projective structures on $C$ is an affine space for the space of quadratic differentials [3]. Let $P$ be a projective structure on $C$ and $\omega \in H^{0}\left(C, K_{C}^{\otimes 2}\right)$ a quadratic differential. Let $(U, z)$ be a coordinate chart compatible with $P$. So the restriction of $\omega$ to $U$ is of the form $h d z \otimes d z$, where $h$ is a holomorphic function. Let $w$ be a function on $U$ such that $\mathcal{S}_{z}(w)=h$, where $\mathcal{S}_{z}$ is the Schwarzian differential operator with respect to $z$ defined by

$$
\mathcal{S}_{z}(f):=\frac{2 f^{\prime}(z) f^{\prime \prime \prime}(z)-3\left(f^{\prime \prime}(z)\right)^{2}}{2\left(f^{\prime}(z)\right)^{2}} .
$$

Then $(U, w)$ is a coordinate chart compatible with the projective structure $P+\omega$ (see [3] for the details).

Let $\overline{\mathcal{F}}$ be a transversely holomorphic structure on the foliation $\mathcal{F}$. The normal bundle

$$
N:=T M / \mathcal{F}
$$

clearly has a structure of a complex line bundle. Indeed, this is an immediate consequence of the condition (3) in Definition 2.1. Therefore, for every integer $k \in \mathbb{Z}$, we have a complex line bundle $N^{\otimes k}$ obtained by taking the $k$ th tensor power of the complex line bundle $N$. By $N^{\otimes-1}$ we mean the dual line bundle $N^{*}$.

The line bundle $N$, and hence any $N^{\otimes k}$, has the Bott partial connection [6]. Recall that the Lie bracket operation on the sheaf of sections of the tangent bundle $T M$ defines the Bott partial connection

$$
N \longrightarrow \mathcal{F}^{*} \otimes N
$$

on $N$ along the foliation $\mathcal{F}$. Since $\mathcal{F}$ is closed under the Lie bracket operation, the composition $[T M, T M] \longrightarrow T M \longrightarrow N$ gives the operator in (2.4). The Jacobi identity for Lie bracket ensures that this partial connection is flat. The partial connection of $N^{\otimes k}$ is induced by the partial connection on $N$.

The conjugate of $N^{*}$ will be denoted by $\overline{N^{*}}$. Over each $U_{i}$ in Definition 2.1, consider $\phi_{i}^{*} \bar{K}_{\mathbb{C}}$, where $\bar{K}_{\mathbb{C}}$ is the space of anti-holomorphic differentials. Denote this line bundle $\phi_{i}^{*} \bar{K}_{\mathbb{C}}$ over $U_{i}$ by $\bar{L}_{i}$. For any pair $U_{i}$ and $U_{j}$, glue $\bar{L}_{i}$ with $\bar{L}_{j}$ over $U_{i} \cap U_{j}$ using the differential $\bar{\partial}\left(\overline{f_{i, j}}\right)$ for the map $f_{i, j}$ in Definition 2.1(3). It is easy to see that these line bundles $\bar{L}_{i}$ patch compatibly to give a line bundle over $M$. The line bundle over $M$ obtained this way clearly coincides with $\overline{N^{*}}$. The Bott partial connection on $N$ induces a partial connection on $\overline{N^{*}}$. 
Any such line bundle $N^{\otimes k}$ has a natural transversely holomorphic structure. This means that there is a first order differential operator

$$
\bar{\partial}_{N \otimes k}: N^{\otimes k} \longrightarrow \overline{N^{*}} \otimes N^{\otimes k}
$$

from the local section of $N^{\otimes k}$ flat under the Bott partial connection to flat sections of $\overline{N^{*}} \otimes N^{\otimes k}$ satisfying the Leibniz identity. For any flat local section $s$ of $N^{\otimes k}$, defined over some open subset of some $U_{i}$ in Definition 2.1, and for any $C^{\infty}$ function $f$ defined on some open subset of $\phi_{i}\left(U_{i}\right)$, the Leibniz identity says

$$
\bar{\partial}_{N \otimes k}\left(\left(f \circ \phi_{i}\right) s\right)=\left(f \circ \phi_{i}\right) \bar{\partial}_{N \otimes k}(s)+\phi_{i}^{*} \bar{\partial} f \otimes s .
$$

The operator $\bar{\partial}_{N \otimes k}$ is simply the Dolbeault operator on the holomorphic tangent bundle $T_{\mathbb{C}}^{\otimes k}$ of the complex line $\mathbb{C}$ transported to $M$ using the projections $\phi_{i}$. It may be noted that the condition in Definition 2.1(3), that every $f_{i, j}$ is holomorphic, ensures that these locally defined operators patch compatibly to define the global differential operator $\bar{\partial}_{N \otimes k}$.

A (local) section $s$ of $N^{\otimes k}$ will be called holomorphic if $\bar{\partial}_{N \otimes k}(s)=0$.

It is easy to see that both the complex structure of $N$ and the transversely holomorphic structure of $N$ are compatible with respect to the Bott partial connection. In other words, both the complex vector space structure of the fibers of $N$ and the Dolbeault operator $\bar{\partial}_{N}$ defined in (2.5) commute with the differential operator in (2.4) defining the Bott connection. Equivalently, parallel translation (for the Bott connection) along the leaves of the foliation $\overline{\mathcal{F}}$ of holomorphic sections of $N$ remain holomorphic. Also, parallel translations for the Bott connection commute with multiplication by $\sqrt{-1}$ of the fibers of $N$.

As we noted, the Bott partial connection on $N$ induces a flat partial connection on any $N^{\otimes k}$. The above compatibility properties of the Bott connection with the complex structure and the holomorphic structure evidently remain valid for any $N^{\otimes k}$.

Consider the space of all globally defined smooth sections over $M$ of the complex line bundle $N^{-2}$ that are flat with respect to the Bott partial connection. Let $\mathcal{V}_{\overline{\mathcal{F}}}$ denote its subspace consisting of all sections that are transversely holomorphic for the transversely holomorphic foliation $\overline{\mathcal{F}}$. In other words, $s \in \mathcal{V}_{\overline{\mathcal{F}}}$ if

$$
\bar{\partial}_{N \otimes-2}(s)=0
$$

for the operator $\bar{\partial}_{N \otimes-2}$ in (2.5). The complex vector space $\mathcal{V}_{\overline{\mathcal{F}}}$ need not be of finite dimension.

Let $\mathcal{P}(\overline{\mathcal{F}})$ denote the space of transversely projective structures on $M$ such that the underlying transversely holomorphic structure coincides with $\overline{\mathcal{F}}$.

Theorem 2.6. The space $\mathcal{P}(\overline{\mathcal{F}})$ is an affine space for the vector space $\mathcal{V}_{\overline{\mathcal{F}}}$.

Proof. Let $\mathcal{P} \in \mathcal{P}(\overline{\mathcal{F}})$ be a transversely projective structure defined by

$$
\left\{U_{i}, \phi_{i}, f_{i, j}\right\}_{i, j \in I}
$$

as in Definition 2.2. Let $\mathcal{P}_{1} \in \mathcal{P}(\overline{\mathcal{F}})$ be another transversely projective structure. Choosing a finer open covering for the data defining $\mathcal{P}$ and $\mathcal{P}_{1}$, we may assume that $\mathcal{P}_{1}$ is defined by the data $\left\{U_{i}, \psi_{i}, g_{i, j}\right\}_{i \in I}$. In other words, the open coverings are the same for both $\mathcal{P}$ and $\mathcal{P}_{1}$. 
For every $i \in I$, we have the commutative diagram of maps

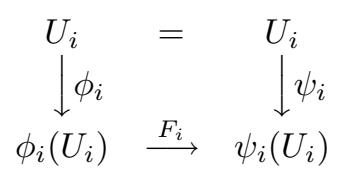

where $F_{i}$ is a biholomorphism. Now, $\psi_{i}\left(U_{i}\right)$ being an open subset of $\mathbb{C}$, it has a canonical projective structure. The pullback of this projective structure, using the isomorphism $F_{i}$, defines a projective structure on $\phi_{i}\left(U_{i}\right)$. This projective structure on $\phi_{i}\left(U_{i}\right)$ will be denoted by $\mathcal{P}_{i, 1}$. On the other hand, since $\phi_{i}\left(U_{i}\right)$ is an open subset of $\mathbb{C}$, it has a natural projective structure. This projective structure on $\phi_{i}\left(U_{i}\right)$ will be denoted by $\mathcal{P}_{i, 0}$.

There is a quadratic differential $\omega_{i}$ on $\phi_{i}\left(U_{i}\right)$, i.e., a holomorphic section of $K_{\mathbb{C}}^{2}$ over $\phi_{i}\left(U_{i}\right)$, such that

$$
\mathcal{P}_{i, 1}=\mathcal{P}_{i, 0}+\omega_{i}
$$

Indeed, as we have already noted, any two projective structures on a Riemann surface differ by a quadratic differential.

The pullback $\phi_{i}^{*} \omega_{i}$ defines a transversely holomorphic section of $N^{\otimes-2}$ over $U_{i}$. This section is evidently flat with respect to the Bott partial connection.

For any $j \in I$, define $\omega_{j}$ on $\phi_{j}\left(U_{j}\right)$ as above. On $U_{i} \cap U_{j}$ the two sections of $N^{\otimes-2}$, defined by $\phi_{i}^{*} \omega_{i}$ and $\phi_{j}^{*} \omega_{j}$ respectively, clearly coincide. In other words, these locally defined sections $\phi_{i}^{*} \omega_{i}$ patch together to give a globally defined section $\omega$ of $N^{-2}$. This section is clearly in $\mathcal{V}_{\overline{\mathcal{F}}}$.

Conversely, if we have a transversely projective structure $\mathcal{P}$ and a holomorphic section $\omega \in \mathcal{V}_{\overline{\mathcal{F}}}$, then using the fact that the space of projective structures on a Riemann surface $C$ is an affine space for the space of quadratic differentials on $C$, it is a straight-forward exercise to construct a new transversely projective structure $\mathcal{P}_{1}$ on $\overline{\mathcal{F}}$. We earlier described the affine space structure of the space of projective structures on a Riemann surface. That construction can be reproduced in the context of transversely holomorphic foliations. In fact, we can just retrace the earlier construction of a section in $\mathcal{V}_{\overline{\mathcal{F}}}$ from a pair of transversely projective structure on $\overline{\mathcal{F}}$.

Finally, it is straight-forward to check that the map

$$
\mathcal{P}(\overline{\mathcal{F}}) \times \mathcal{V}_{\overline{\mathcal{F}}} \longrightarrow \mathcal{P}(\overline{\mathcal{F}})
$$

defined by $(\mathcal{P}, \omega) \longmapsto \mathcal{P}_{1}$ makes $\mathcal{P}(\overline{\mathcal{F}})$ an affine space for the vector space $\mathcal{V}_{\overline{\mathcal{F}}}$. This completes the proof of the theorem.

We will now specialize to the situation where the manifold $M$ is a compact Kähler manifold. Furthermore, $\mathcal{F}$ is assumed to be a holomorphic subbundle of the holomorphic tangent bundle $T_{M}$ of $M$. In other words, $\mathcal{F}$ is a holomorphic foliation of codimension one on the complex manifold $M$. In particular, $\mathcal{F}$ has an induced structure of a transversely holomorphic foliation. So, the maps $\phi_{i}$ in Definition 2.1 are taken to be holomorphic.

Let $P(\mathcal{F})$ denote the space of all transversely projective structures on $\mathcal{F}$ satisfying the following two conditions:

(1) for any $P \in P(\mathcal{F})$, its underlying transversely holomorphic foliation coincides with $\mathcal{F}$; 
(2) $P$ is holomorphic, or in other words, the maps $\phi_{i}$ in Definition 2.2 are holomorphic submersions.

From Theorem 2.6 it follows that $P(\mathcal{F})$ is an affine space for the space of all holomorphic sections of $N^{\otimes-2}$ that are flat with respect to the Bott partial connection.

We recall the definition of semistability of a vector bundle over a compact Kähler manifold [5. Fix a Kähler form $\beta$ on $M$. A vector bundle $E$ over $M$ is called semistable if for any coherent analytic subsheaf $F \subset E$ of positive rank, the inequality

$$
\frac{\operatorname{degree}(F)}{\operatorname{rank}(F)} \leq \frac{\operatorname{degree}(E)}{\operatorname{rank}(E)}
$$

is valid. The degree of a coherent sheaf $F$ is defined as

$$
\operatorname{degree}(F)=\int_{M} c_{1}(F) \beta^{\operatorname{dim} M-1}
$$

where $\operatorname{dim} M$ is the complex dimension of $M$. Note that $c_{1}(F) \beta^{\operatorname{dim} M-1}$ is a top degree cohomology class of $M$.

Lemma 2.7. Let the holomorphic tangent bundle $T_{M}$ be semistable of positive degree. Then given any holomorphic foliation $\mathcal{F}$ of codimension one, there is at most one transversely projective structure on $\mathcal{F}$. In other words, the cardinality of the set $P(\mathcal{F})$ is not more than one.

Proof. From Theorem 2.6 it follows that if $P, P^{\prime} \in P(\mathcal{F})$, then the difference $P^{\prime}-P$ is a holomorphic section of the holomorphic line bundle $N^{\otimes-2}$ over $M$. Therefore, it suffices to show that $N^{\otimes-2}$ does not admit any nonzero holomorphic section.

Since $N$ is a quotient bundle of $T_{X}$, the semistability condition ensures that the degree of $N$ is positive. Hence degree $\left(N^{\otimes-2}\right)=-2 \operatorname{degree}(N)$ is negative. Therefore, we have

$$
H^{0}\left(M, N^{\otimes-2}\right)=0
$$

since any semistable vector bundle of negative degree does not admit any nonzero section. This completes the proof of the lemma.

Now assume that $M$ is a complex projective manifold with $\operatorname{dim} M \geq 2$. We further assume that

$$
\mathrm{NS}(M):=H^{1,1}(M) \cap H^{2}(M, \mathbb{Z})=\mathbb{Z},
$$

where $H^{1,1}$ corresponds to the Hodge decomposition of $H^{2}(M, \mathbb{C})$. The group $\mathrm{NS}(M)$ is known as the Neron-Severi group.

An immediate consequence of the assumption on $\mathrm{NS}(M)$ is that if $L$ is a holomorphic line bundle over $M$ with $c_{1}(L) \neq 0$, then for any other line bundle $L^{\prime}$ over $M$, the first Chern class $c_{1}\left(L^{\prime}\right)$ is a scalar multiple of $c_{1}(L)$.

Lemma 2.8. $\operatorname{dim} P(\mathcal{F}) \leq 1$.

Proof. Fix an ample class $\omega_{0} \in \mathrm{NS}(M)$. So $\omega_{0}$ is represented by a positive $(1,1)$ form (also called a Kähler form). Let $c \in \mathbb{Q}$ be such that

$$
c_{1}(N)=c \omega_{0} .
$$

The existence of such a scalar is ensured by the assumption that $\operatorname{NS}(M)=\mathbb{Z}$. 
A theorem of Bott says that $c_{1}(N)^{2}=0$ [6, Theorem 3.4], [1]. So we have

$$
c^{2}\left(\omega_{0}\right)^{2}=0 \text {. }
$$

Now, since the cohomology class $\omega_{0}$ is ample and $\operatorname{dim} M \geq 2$, we have $\left(\omega_{0}\right)^{2} \neq 0$. Therefore, $c=0$. In other words, we have $c_{1}(N)=0$. Consequently, $N$ is topologically trivial. This implies that

$$
\operatorname{dim} H^{0}(M, N) \leq 1
$$

Indeed, if $\operatorname{dim} H^{0}(M, N) \geq 2$, then given any two linearly independent sections of $N$, say $s_{1}$ and $s_{2}$, some linear combination of them, say $s=\lambda_{1} s_{1}+\lambda_{2} s_{2}$, vanishes somewhere but it is not identically zero. Let $\theta \in H^{2}(M, \mathbb{Z})$ be the cohomology class represented (using Poincaré duality) by the divisor of $M$ defined by $s$. Since $\theta \neq 0$ and $\theta=c_{1}(N)$, we have a contradiction. This establishes (2.9).

In view of Theorem 2.6, the inequality $(2.9)$ implies that $\operatorname{dim} P(\mathcal{F}) \leq 1$. This completes the proof of the lemma.

A topologically trivial holomorphic line bundle admits a nonzero section if and only if the line bundle is holomorphically trivial. Therefore, in Lemma 2.8 we have $\operatorname{dim} P(\mathcal{F})=1$ if and only if $N$ is holomorphically trivial and the trivialization of $N$ is flat with respect to the Bott partial connection on $N$.

\section{REFERENCES}

[1] R. Bott, On a topological obstruction to integrability, "Global Analysis" (Proc. Sympos. Pure Math., Vol. XVI, Berkeley, Calif., 1968) 127-131. MR 42:1155

[2] C. Godbillon, "Feuilletages études géométriques", Progress in Math., vol. 98, Birkhäuser, 1991. MR 93i:57038

[3] R.C. Gunning, "Lectures on Riemann surfaces", Mathematical Notes 2, Princeton University Press, Princeton, New Jersey 1966. MR 34:7789

[4] A. Haefliger, Homotopy and integrability. "Manifolds-Amsterdam 1970", Proc. Nuffic Summer School on Manifolds (Ed. N. H. Kuiper) pp. 133-163, Lecture Notes in Mathematics, Vol. 197, Springer, Berlin, 1971. MR 44:2251

[5] S. Kobayashi, "Differential Geometry of Complex Vector Bundles", Publications of the Math. Society of Japan 15, Iwanami Shoten Publishers and Princeton University Press, 1987. MR 89e:53100

[6] H.B. Lawson, "The quantitative theory of foliations", CBMS Regional Conference Series in Math., No. 27, American Mathematical Society, 1977.

School of Mathematics, Tata Institute of Fundamental Research, Homi Bhabha ROAD, BOMBAY 400005, INDiA

E-mail address: indranil@math.tifr.res.in 